\title{
Cholelithiasis after Weight Loss Surgery: Challenge and Prophylaxis
}

\author{
Ahmed Talha MD, MRCS; ${ }^{1}$ Ayman Farouk, MD, ${ }^{1}$ Ihab Hasouna, MD. ${ }^{2}$
}

1) Department of Surgery, Medical Research Institute, Alexandria University, Egypt.

2) Department of Internal Medicine, Faculty of Medicine, Alexandria University, Egypt.

Background: Rapid loss of weight after bariatric surgery is associated with a high incidence of gallstone formation. This study was carried out to detect the efficacy of six months regimen of prophylactic Ursodeoxycholic acid in the prevention of gallstones and to identify the predictive factors for gallstone formation after weight loss surgery.

Methods: A randomized controlled trial was carried out involving 108 patients with a preoperative diagnosis of morbid obesity were subjected to either laparoscopic sleeve gastrectomy (LSG) or greater curve plication (LGCP) with follow-up for a minimum one year; they were divided into two groups; (group A; receiving Ursodeoxycholic acid) and (group B; receiving placebo). Data were collected about: Patient clinical history, baseline characteristics and postoperative follow-up.

Results: The demographic parameters were comparable in the two groups. The incidence of cholelithiasis after surgery was $14.3 \%$ (13 cases). The mean $\%$ EWL was significantly higher in those who develop gallstones than others $(P=0.045)$. Also, there was a significant increased cholelithiasis post-LSG than LGCP $(P=0.036)$. There was a significant decrease in the incidence of gallstone formation from $22 \%$ in placebo to $6.5 \%$ in treated group with Ursodeoxycholic $\operatorname{acid}(P=0.041)$.

Conclusions: The percentage of excess weight loss was the only predictive postoperative factor for gallstone formation. A six months use of Ursodeoxycholic acid is an effective prophylaxis for gallstone formation after weight loss procedures although a larger study is required to reach a definitive conclusion.

Key Words: Obesity; gallstone; bariatric surgery; ursodeoxycholic acid.

\section{Introduction:}

Worldwide prevalence of obesity is increasing, and the incidence of obesity in the US has increased from $22.9 \%$ between 1988 and 1994 , to $34 \%$ in $2006 .^{1-3}$

Obesity is associated with increased risk for hypertension, diabetes, pulmonary disease, hyperlipidemia, cardiomyopathy, malignancy, arthritis, infertility, sleep apnea, gallstone formation and psychosocial impairments. Given the fact that with weight loss improves many of these comorbidities, ${ }^{4}$ much effort has gone into the development of effective treatment modalities focused on sustained weight loss. Dietary regimen, behavioral modification, and exercise have been largely unsuccessful in achieving and maintaining long-term results in morbidly obese patients. Therefore, more aggressive treatment is typically required for obese subjects at risk for medical complications of obesity. Surgery has become an attractive alternative because it represents a long-term solution. ${ }^{5}$

Bariatric surgery is the most effective modality for long-term weight loss and for resolving the associated comorbidities. ${ }^{6}$ The 
primary mechanisms through which bariatric surgery achieves its outcomes are believed to be the mechanical restriction of food intake, reduction in the absorption of ingested foods, or a combination of both. ${ }^{7}$ However, controversies exist regarding the ideal weight loss metabolic procedure that allowed continuous search for new techniques.

Many operative modalities have been devised, among those most commonly performed nowadays; adjustable gastric banding (AGB); Roux-en-Y gastric bypass (RYGB); biliopancreatic diversion with duodenal switch (BPD); and sleeve gastrectomy (SG) and greater curve plication (GCP) preferably through minimally invasive approach. ${ }^{5-8}$

Between 35-38\% of patients with morbid obesity develop gallstones as they lose weight after bariatric surgery.5,9-11 A routine synchronous cholecystectomy during bariatric surgery is recommended by some centers.9,12 Therefore, a preventive therapy for gallstone formation is recommended in several studies. Ursodeoxycholic acid (500 mg/d) is highly effective in preventing gallstone formation in patients undergoing dietary-induced weight reduction. ${ }^{13}$

This study was carried out to detect the efficacy of six months regimen of prophylactic Ursodeoxycholic acid in the prevention of gallstones and to identify the predictive factors for gallstone formation after weight loss surgery.

\section{Study design:}

This was randomized controled trial study, where all patients from age 18 to 60 years, with a preoperative diagnosis of morbid obesity based on the guidelines issued by International Federation for Surgery of Obesity (IFSO) ${ }^{14}$ underwent either laparoscopic sleeve gastrectomy (LSG) or laparoscopic greater curve plication (LGCP) at the Department of Surgery, Medical Research Institute Hospital, Alexandria University and continued their follow-up for a minimum of one year. 108 patients were divided into two groups; (group A; receiving Ursodeoxycholic acid; $500 \mathrm{mg} / \mathrm{d}$ for six months in the immediate postoperative period) and (group B; receiving placebo), were offered the opportunity to participate in this trial. Informed consent was obtained from all participants and approval was obtained from the ethics committee of our institutions.

The exclusion criteria included the following: American Society of Anesthesiologists (ASA) class IV and V, patients with contraindication for laparoscopy, prior cholecystectomy, presence of gallstones, use of other investigational drugs, pregnancy or refusal.

\section{Randomization method:}

Eligible patients were randomized into two groups; (group A; receiving Ursodeoxycholic acid) and (group B; receiving placebo) using sealed opaque envelopes containing computer-generated random numbers. The randomization was performed one week before surgery during the preoperative assessment. Data were collected about: Patient clinical history, baseline characteristics and postoperative follow-up evaluating the percentage of excess weight loss (\% EWL) and gallstone formation.

\section{Study Protocol:}

All patients underwent the following basic preoperative investigations, including the following: (1) blood tests: complete blood count, coagulation profile, renal and liver function tests and hormonal profile including TSH and cortisone levels; (2) radiologic imaging: chest radiograph and ultrasonography of abdomen and pelvis; (3) electrocardiogram; (4) echocardiogram; and (5) respiratory function tests.

Eligible patients had undergone either LSG or LGCP. Ambulation was encouraged, and chest physiotherapy was started in the immediate postoperative period. An upper gastrointestinal contrast study using watersoluble contrast (Gastrograffin) was done on the first postoperative day. Clear liquids were started on confirmation of staple-line integrity. The patient was discharged once oral intake of 1,500-2,000 ml/24 h was established. Prokinetics, and proton pump inhibitors were continued for ten days. All 
medications were given orally in crushed or liquid form. The drain was removed on the second postoperative day. A liquid diet was given for two weeks, a pureed/soft diet for six weeks, and normal diet thereafter. Dietary counseling was provided, and a normal consistency, low-calorie, high protein diet is advised at two months from surgery. Patients were followed up at 1, 3, 6, and 12 postoperative months and then annually. Abdominal Ultrasonography investigation of the gallbladder was performed in all patients at six and twelve postoperative months.

\section{Statistical analysis:}

Statistical analysis was performed using the Statistical Package for the Social Sciences (SPSS) version 20 software (SPSS, Inc., Chicago, IL, USA). Significance was set at a P-value <0.05. Qualitative data were described using number and percent. Quantitative data were described using mean and standard deviation for normally distributed data. Comparison between different groups regarding categorical variables was tested using Chi-square test while for two groups comparison t-test was used for parametric data.

\section{Results:}

From March 2010 to October 2013, 134 patients were submitted to bariatric surgery and 108 patients were eligible. 91 patients completed follow-up gallbladder sonography and were randomly classified into two groups; 45 to placebo and 46 to $500 \mathrm{mg} / \mathrm{d}$ Ursodeoxycholic acid. Twenty six patients refused to participate in the study or did not meet the inclusion criteria. (Figure I) 66 patients (72.5\%) patients underwent LSG and 25 patients (27.5\%) underwent LGCP Table (1).

There were 36 men (39.6\%) and 55 women (60.4\%). There were no significant differences with respect to age, sex, or preoperative BMI between those who developed gallstones or not. Table (1) However, the group who formed gallstones have significantly higher mean \% EWL in the first postoperative year $(\mathrm{P}=0.045)$. Table (1) Regarding the type of operation there was a significant difference between those who developed gallstones or not $(\mathrm{P}=0.036)$ as in LSG $10.9 \%$ versus $3.3 \%$ in LGCP; patients showed a noticeably more rapid weight loss, with significant incident development of gallstone formation Table (1).

Ultrasonography investigation of the gallbladder was performed in all patients. During follow up, 13 of them (14.3\%) developed gallstones postoperatively in a range of 6 - 12 months and 78 (85.7\%) did not develop gallstones Table (1). Of those developed gallstones 5 cases (38.5\%) were symptomatizing and the other 8 cases (61.5\%) discovered by routine ultrasonography during follow up. So the incidence of symptomatizing cholelithiasis after surgery in this series was $5.5 \%$ Table (2).

There were no significant differences with respect to age, sex, preoperative BMI, $\% \mathrm{EWL}$ or the type of surgery between those receiving medication and placebo in any of the patient groups. Table (3).

No patient was withdrawn from the study because of a serious adverse drug reaction. No severe side effects from medication were observed. Mild and moderate side effects such as nausea and constipation were equivalent in both groups

Gallstone formation was significantly less frequent $(\mathrm{P}=0.041)$ with Ursodeoxycholic acid than with placebo at 12 months: 6.5\% versus 22\%, respectively. Table (2). 5 cholecystectomies were performed (mean $14.9 \pm 4.3$ ) months after surgery in patients with symptomatic cholelithiasis: 1 patient in the Ursodeoxycholic acid group and 4 patients in the placebo group, $2 \%$ versus 8.9 $\%$, respectively. ( $\mathrm{P}=0.11$ ) Table (2).

\section{Discussion:}

Obesity and rapid weight loss are well known risk factors for cholelithiasis as approximately one third of patients may develop gallstones after bariatric surgery. Furthermore, $10 \%$ to $15 \%$ of all patients will require cholecystectomy for complaints related to gallstones. ${ }^{9-13}$ Some centers routinely perform cholecystectomies with bariatric procedures to prevent 
complications of cholelithiasis, prophylactic cholecystectomy is not preferred as the operation may increase the overall operative time and length of hospital stay. In addition, a cholecystectomy after losing weight may be technically easier than during maximum obesity. ${ }^{11-13}$ Also, Angrisani et al ${ }^{15}$ reported that laparoscopic cholecystectomy in obese patients was technically more difficult, and required a significantly longer operating time. In the present study, we excluded patients who had previous cholecystectomy or requiring concomitant cholecystectomy to avoid the debate about this difficulty and the prolonged operative time.

The risk of developing gallstones in obese patients is increased that could be due to a higher cholesterol saturation of gallbladder bile, diminished gallbladder motility with subsequent stasis and increased levels of gallbladder mucin promoting precipitation of cholesterol crystals ${ }^{16}$ During rapid weight loss produced by weight loss surgery with very low caloric diet, the incidence of gallstone formation increased, ${ }^{17}$ but the underlying mechanism is not fully understood, and some pathogenic mechanisms have been proposed including: increased cholesterol saturation index of bile as a result of cholesterol mobilization from adipose tissues and excretion in bile, increased gallbladder secretion of mucin and calcium, and increased presence of prostaglandins and arachidonic acid. ${ }^{16,18-21}$

The present study revealed five out of thirteen cases who developed gallstones were symptomatic with the overall symptomatic gallstone incidence after surgery $5.5 \%$ which is lower than the findings as Tucker et al. 22 reported incidence of $6 \%$, Portenier et $\mathrm{al}^{23} 8.1 \%$, Papasavas et $\mathrm{al}^{24} 6.9 \%$, Villegas et al $257.3 \%$ while Abo-Ryia et al ${ }^{26}$ reported $8 \%$. This may be because we had two groups one was taking Ursodeoxycholic acid with a lower incidence of gallstone formation, thus, decreasing the overall number of gallstone incidence.

Weight loss after antiobesity surgery is maximal during the first postoperative year, decreasing over time. The peak of symptomatic gallstone disease at 2 years after surgery. $.27,28$

The higher occurrence of cholelithiasis following weight reduction surgery was in the phase of greater weight loss that encouraged surgeons to use Ursodeoxycholic acid as prophylaxis in the first 6 postoperative months. 9,29

This drug is a bile acid that prevents biliary lithiasis by decreasing cholesterol and mucin concentration, increasing bile acid concentration, decreasing bile saturation, and enhancing gallbladder emptying. ${ }^{9,29,30}$

Two controlled trials ${ }^{31,32}$ have shown the effectivenessofsixmonthsofUrsodeoxycholic acid treatment in lowering the incidence of gallstone formation. Cholelithiasis was found in 22-32\% of controls versus in 2- $3 \%$ of treated patients at 6-12 months. Similarly, we found a significant decrease in the incidence of cholelithiasis after bariatric surgery from $22 \%$ in placebo versus $6.5 \%$ in patients receiving Ursodeoxycholic acid for six months in the immediate postoperative period.

Age, obesity, female gender and parity are known risk factors for gallstones formation of are known, in the general population which is most likely due to the female sex hormones. ${ }^{33}$ We tried to identify the risk factors for cholelithiasis after weight loss surgery by comparing the patients who developed gallstones during rapid weight loss with those who did not. In our study, Age, gender and mean preoperative BMI were not significant between both groups so, not considered as predictors for gallstone formation which is similar to that reported by others. ${ }^{26,27,34}$ However the mean percent of excess weight loss in the first postoperative year was significantly higher in the group that formed gallstone which is in accordance to the findings of Schmidt et al. ${ }^{35}$ Ming Li et al. ${ }^{34}$ Wudel et al. ${ }^{30}$ Yang et al. ${ }^{28}$ and AboRyia et $\mathrm{al}^{26}$ In another study, only weight loss of more than $25 \%$ was associated with an increased risk of gallstone disease after antiobesity surgery. 35

It is accepted that gallstone formation after bariatric surgery is related to weight loss. ${ }^{9}$ 




Figure (1): Trial flow sheet showing progress through the phases of the trial.

Table (1): Predictors of cholelithiasis in either group.

\begin{tabular}{|c|c|c|c|}
\hline Variable & Gallstone & No gallstone & P-value \\
\hline No (\%) & $13(14.3 \%)$ & $78(85.7 \%)$ & $0.001 *$ \\
\hline Mean age in years & $31.2 \pm 8.11$ & $30.8 \pm 7.65$ & 0.852 \\
\hline $\begin{array}{ll}\text { Gender } & \text { Male } \\
& \text { Female }\end{array}$ & $\begin{array}{l}5(5.5 \%) \\
8(8.8 \%)\end{array}$ & $\begin{array}{l}31(34 \%) \\
47(51.6 \%)\end{array}$ & $\begin{array}{l}0.011^{*} \\
0.003^{*}\end{array}$ \\
\hline $\begin{array}{l}\text { Mean preoperative } \\
\mathrm{BMI} \text { in } \mathrm{kg} / \mathrm{m}^{2}\end{array}$ & $48.2 \pm 11.25$ & $49.1 \pm 12.1$ & 0.521 \\
\hline $\begin{array}{l}\text { Mean \% EWL in the } \\
\text { first year }\end{array}$ & $26.2 \pm 8.3$ & $23.1 \pm 9.22$ & $0.045^{*}$ \\
\hline $\begin{array}{c}\text { Type of operation } \\
\text { LSG } \\
\text { LGCP }\end{array}$ & $\begin{array}{l}10(10.9 \%) \\
3(3.3 \%) \\
P=0.036^{*}\end{array}$ & $\begin{array}{l}56(61.5 \%) \\
22(24.2 \%) \\
P=0.236\end{array}$ & $\begin{array}{l}0.012 * \\
0.002 *\end{array}$ \\
\hline
\end{tabular}

BMI; body mass index, SD; standard deviation, \%EWL; percentage of excess weight loss

Shiffman et $\mathrm{al}^{36}$ showed that bile cholesterol normalized when the weight stabilized 24 months after gastric bypass. Miller et $\mathrm{al}^{37}$ demonstrate in their prospective study on more than 1000 restrictive procedures that a weight stabilization phase is reached not before 24 months and in another study, a significantly reduced gallstone formation rate of $8 \%$ after 24 months compared with $30 \%$ in the placebo group. ${ }^{9}$ According to Shiffman et al, at 6 months, gallstones had developed in $36 \%$ and gallbladder sludge in additional $13 \%$ of patients. ${ }^{36}$ We reported 13 patients (14.3\%) out of 91 had gallstones at one year and this short follow-up period could be an explanation of smaller percentage of gallstone formation in our patients.

The bariatric procedure used to achieve 
Table 2: Distribution of patients free of gallstone at the time of surgery.

\begin{tabular}{|l|l|l|l|l|l|l|}
\hline \multirow{2}{*}{ Gallstone formation after weight loss } & \multicolumn{3}{|l|}{ Group “A” (n=46) } & Group “B” (n=45) & \multirow{2}{*}{ P-value } \\
\cline { 3 - 7 } & No & $\%$ & No & $\%$ & \\
\hline \multirow{2}{*}{ Developed gallstones } & Symptomatic & 1 & 2.2 & 4 & 8.9 & 0.11 \\
\cline { 2 - 7 } & Asymptomatic & 2 & 4.3 & 6 & 13.3 & 0.365 \\
\hline & Total & 3 & 6.5 & 10 & 22.0 & $0.041^{*}$ \\
\hline \multirow{2}{*}{ Did not develop gallstones } & 43 & 93.5 & 35 & 77.8 & 0.25 \\
\hline Total & 46 & 45 & & \\
\hline
\end{tabular}

Table (3): Demographic data of both groups

\begin{tabular}{|l|l|l|l|}
\hline Variable & Group “A” $(\mathrm{n}=46)$ & Group “B” (n=45) & P-value \\
\hline Mean age in years & $31.08 \pm 9.01$ & $30.1 \pm 7.01$ & 0.465 \\
\hline $\begin{array}{l}\text { Gender Male } \\
\text { Female }\end{array}$ & $\begin{array}{l}17(37.0 \%) \\
29(63.0 \%)\end{array}$ & $\begin{array}{l}19(42.2 \%) \\
26(57.8 \%)\end{array}$ & 0.365 \\
\hline $\begin{array}{l}\text { Mean preoperative } \\
\text { BMI in kg/m² }\end{array}$ & $48.6 \pm 10.6$ & $49.3 \pm 12.6$ & 0.622 \\
\hline $\begin{array}{l}\text { Mean \% EWL in the } \\
\text { first year }\end{array}$ & $25.3 \pm 7.11$ & $24.9 \pm 10.1$ & 0.366 \\
\hline $\begin{array}{c}\text { Type of operation } \\
\text { LSG } \\
\text { LGCP }\end{array}$ & $33(71.7 \%)$ & $33(73.3 \%)$ & 0.698 \\
$13(28.3 \%)$ & $12(26.7 \%)$ & 0.71 \\
\hline
\end{tabular}

weight loss could influence the risk of developing symptomatic gallstone disease. ${ }^{37}$ Patients who had undergone gastric bypass were at a greater risk than those who had undergone a restrictive procedure. ${ }^{37}$ Previous studies have demonstrated similar rates of cholecystectomy after gastric bypass and gastric banding $(8.1 \%$ and $6.8 \%$, respectively) ${ }^{38-40}$ In our study, we found a significant difference between both groups as the incidence of gallstone formation was higher in LSG than in LGCP while, Ming Li et al. ${ }^{34}$ reported insignificant difference regarding the type of operation; Gastric Bypass, Gastric Banding, and Sleeve Gastrectomy.

\section{Conclusion:}

The percentage of excess weight loss was the only predictive postoperative factor for gallstone formation. A six months use of Ursodeoxycholic acid is an effective prophylaxis for gallstone formation after weight loss procedures although a larger study is required to reach a definitive conclusion.

Conflict of Interest: There is no conflict of interest or financial ties to include.

\section{Reference:}

1- Rennie KL, Jebb SA: Prevalence of obesity in Great Britain. Obes Rev 2005; 6: 11-12.

2- Flegal KM, Carroll MD, Ogden CL, et al: Prevalence and trends in obesity among US adults, 1999-2000. JAMA 2002; 288: 1723-1727.

3- Klein S, Wadden T, Sugerman HJ: AGA technical review on obesity. Gastroenterology 2002; 123: 882-932.

4- Pi-Sunyer FX: A review of long-term studies evaluating the efficacy in weight loss ameliorating disorders associated with obesity. Clin Ther 1996; 18: 1006-1035.

5- Balsiger BM, Murr MM, Poggio JL, Sarr MG: Bariatric surgery. Surgery for weight control in patients with morbid obesity. Med Clin North Am 2000; 84: 77-489.

6- Buchwald H, Avidor Y, Braunwald E, et al: Bariatric surgery: A systematic review and meta-analysis. JAMA 2004; 292(14):1724-1737. 
7- De Maria EJ: Bariatric surgery for morbid obesity. $N$ Engl J Med 2007; 356(21): 2176-2183.

8- Brody F: Minimally invasive surgery for morbid obesity. Cleveland Clinic J Medicine 2004; 71(4): 289-298.

9- Miller K, Hell E, Lang B, Lengauer E: Gallstone formation prophylaxis after gastric restrictive procedures for weight loss: A randomized double-blind placebo-controlled trial. Ann Surg 2003; 238: 697-702.

10- Shiffman ML, Sugerman HJ, Kellum JM, Moore EW: Changes in gallbladder bile composition following gallstone formation and weight reduction. Gastroenterology 1992; 103: 214-221.

11- Amaral JF, Thompson WR: Gallbladder disease in the morbidly obese. Am J Surg 1985; 149: 551-557.

12- Deitel M, Petrov I: Incidence of symptomatic gallstones after bariatric operations. Surg Gynecol Obstet 1987; 164: 549-552.

13- Wattchow DA, Hall JC, Whiting MJ, Bradley B, Iannos J, Watts JM: Prevalence and treatment of gallstones after gastric bypass surgery for morbid obesity. Br Med J (Clin Res Ed) 1983; 286: 763.

14- International Federation for the Surgery of Obesity: Statement on patient selection for bariatric surgery. Obes Surg 1997; 7: 41.

15- Angrisani L, Lorenzo M, De Palma G, et al: Laparoscopic cholecystectomy in obese patients compared with nonobese patients. Surg Laparosc Endosc 1995; 5: 197-201.

16- Festi D, Colecchia A, Larocca A, et al: Review: Low caloric intake and gall-bladder motor function. Aliment Pharmacol Ther 2000; 14(2): 51-53.

17- Everhart JE: Contributions of obesity and weight loss to gallstone disease. Ann Intern Med 1993; 119: 1029-1035.

18- Shiffman ML, Shamburek RD, Schwartz $\mathrm{CC}$, et al: Gallbladder mucin, arachidonic acid, and bile lipids in patients who develop gallstones during weight reduction. Gastroenterology. 1993; 105: 1200-1208.

19- Shiffman ML, Sugerman HJ, Kellum JH, et al: Gallstones in patients with morbid obesity. Relationship to body weight, weight loss and gallbladder bile cholesterol solubility. Int $J$ Obes Relat Metab Disord 1993; 17: 153-158.

20- Mason EE: Gallbladder management in obesity surgery. Obesity Surgery 2002; 12(2): 222-229.

21- Dittrick GW, Thompson JS, Campos D, et al: "Gallbladder Pathology in Morbid Obesity,"
Obesity Surgery 2005; 15(2): 238-242.

22- Tucker ON, Fajnwaks P, Szomstein S, et al: Is concomitant cholecystectomy necessary in obese patients undergoing laparoscopic gastric bypass surgery? Surgical Endoscopy 2008; 22(11): 2450-2454.

23- Portenier DD, Grant JP, Blackwood HS, et al: Expectant management of the asymptomatic gallbladder at Roux-en-y gastric bypass. Surgery for Obesity and Related Diseases 2007; 3(4): 476-479.

24- Papasavas PK, Gagné DJ, Ceppa FA, et al: Routine gallbladder screening not necessary in patients undergoing laparoscopic Rouxen-y gastric bypass. Surgery for Obesity and Related Diseases 2006; 2(1): 41-46.

25- Villegas L, Schneider B, Provost D, et al: Is routine cholecystectomy required during laparoscopic gastric bypass? Obesity Surgery 2004; 14(2): 206-211.

26- Abo-Ryia MH, Abd-Allah HS, El-Khadrawy $\mathrm{OH}$, Moussa GI: Predictors of gallstone formation in morbidly obese patients after bariatric surgery: A retrospective observational study. Surg Science 2014; 5: $1-5$.

27- Oliveira CIB, Chaim EA, Silva BB: Impact of rapid weight reduction on risk of cholelithiasis after bariatric surgery. Obesity Surgery 2003; 13(4): 625-628.

28- Yang H, Peterson GM, Roth MP, Schoenfield LJ, Marks JW: Risk factors for gallstones formation during rapid loss of weight. Digestive Diseases and Sciences 1992; 37(6): 912-918.

29- Uy MC, Talingdan-Te MC, Espinosa WZ, et al: Ursodeoxycholic acid in the prevention of gallstone formation after bariatric surgery: A meta-analysis. Obesity Surgery 2008; 18(12): 1532-1538.

30- Wudel LJ, Wright JK, Debelak JP, et al: Prevention of gallstone formation in morbidly obese patients undergoing rapid weight loss: Results of a randomized controlled pilot study. Journal of Surgical Research 2002; 102(1): 50-56.

31- Miller K, Hell E, Lang B, Lengauer E: Gallstone formation prophylaxis after gastric restrictive procedures for weight loss: A randomized double-blind placebo-controlled trial. Ann Surg 2003; 238: 697-702.

32- Sugerman HJ, Brewer WH, Shiffman ML, et al: A multicenter, placebo-controlled, randomized, double-blind, prospective trial of prophylactic ursodiol for the prevention of gallstone formation following 
gastric-bypass-induced rapid weight loss. Am J Surg 1995; 169: 91-96; discussion 96-97.

33- Cirillo DJ, Wallace RB, Rodabough RJ, et al: Effect of estrogen therapy on gallbladder disease. JAMA 2005; 293: 330-339.

34- Ming VK, Pulido LN, Fajnwaks P, et al: Predictors of gallstone formation after bariatric surgery: A multi- variate analysis of risk factors comparing gastric bypass, gastric banding, and sleeve gastrectomy. Surgical Endoscopy 2009; 23(7): 1640-1644.

35- Li VK, Pulido N, Fajnwaks P, Szomstein S, Rosenthal R, Martinez-Duartez P: Predictors of gallstone formation after bariatric surgery: A multivariate analysis of risk factors comparing gastric bypass, gastric banding, and sleeve gastrectomy. Surg Endosc 2009; 23(7): 1640-1644.

36- Shiffman ML, Sugerman HJ, Kellum JM, Brewer WH, Moore EW: Gallstone formation after rapid weight loss: A prospective study in patients undergoing gastric bypass surgery for treatment of morbid obesity. Am $J$ Gastroenterol 1991; 86(8): 1000-1005.

37- Jonas E, Marsk R, Rasmussen F, Freedman J: Incidence of postoperative gallstone disease after antiobesity surgery: Population-based study from Sweden. Surg Obes Relat Dis 2010; 6: 54-58.

38- Miller K, Höller E, Hell E: Restrictive procedures in the treatment of morbid obesity - vertical banded gastroplasty vs. adjustable gastric banding. Zentralbl Chir 2002; 127: 1038-1043.

39- Portenier DD, Grant JP, Blackwood HS, Pryor A, McMahon RL, DeMaria E: Expectant management of the asymptomatic gallbladder at Roux-en-Y gastric bypass. Surg Obes Relat Dis 2007; 3: 476-479.

40- O'Brien PE, Dixon JB: A rational approach to cholelithiasis in bariatric surgery: Its application to the laparoscopically placed adjustable gastric band. Arch Surg 2003; 138: 908-912. 